\title{
Prediction of the Hardness of X12m Using Barkhausen Noise and Chebyshev Polynomials Regression Methods
}

\author{
Zibo $\mathrm{Li}^{1,2,3^{*}}$, Shicheng $\mathrm{Li}^{1,2}$, Donghao Wang ${ }^{1,2}$, Guangmin $\mathrm{Sun}^{3}, \mathrm{Cunfu} \mathrm{He}^{4}, \mathrm{Yu} \mathrm{Li}^{3}$, \\ Xiucheng $\mathrm{Liu}^{4}$, Yanchao $\mathrm{Cai}^{4}$, Chu Wang ${ }^{1,2}$ \\ ${ }^{I}$ Beijing JingHang Research institute of computation and communication, Beijing, \\ 100074, China.Email glory0117@163.com \\ ${ }^{2}$ The Classified Information Carrier Safety Management Engineering Technology \\ Research Center of Beijing, 100074, China \\ ${ }^{3}$ Faculty of Information Technology, Beijing University of Technology, Beijing, \\ 100124, China \\ ${ }^{4}$ College of Mechanical Engineering and Applied Electronics Technology, Beijing \\ University of Technology, Beijing, 100124, China
}

\begin{abstract}
Barkhausen noise (BN) is electromagnetic pulse sequence that could be used to nondestructively predict the properties of materials such as hardness, residual stress and carbon content. Current $\mathrm{BN}$ signal analysis methods fail to describe the highly variated BN signal and achieve high regression accuracy due to the low interpretability of neural network and limited capacity of mathematical regression tools. In this paper, two multi-variable regression tools, named partial Chebyshev polynomial regression (PCPR) and Mutual Information-based Feature Selection with Class-dependent Redundancy and multi-variable Chebyshev polynomials regression (MIFS-CR+MCPR), are employed for the first time to predict the hardness of $\mathrm{Cr} 12 \mathrm{MoV}$ steel (i.e. X12m). Combined with Chebyshev polynomials, our regression tools are designed on the basis of cascaded regression and mutual-information-based feature selection. As represented by the experimental results for predicting the hardness of X12m, the proposed method outperforms other comparative methods including neural network and partial linear square regression method.
\end{abstract}

Keywords. Non-destructive Evaluation, Hardness Prediction, Chebyshev polynomials regression, Barkhausen Noise

\section{Introduction}

As one of the famous non-destructive evaluation (NDE) ${ }^{[1,2]}$ methods, Barkhuasen Noise (BN) is generated by the irreversible movements of domain walls and could be analyzed to predict the properties of materials such as hardness, residual stress and carbon content. The biggest challenge in analyzing $\mathrm{BN}$ is the pseudo-random nature. Therefore, features directly extracted from $\mathrm{BN}$ signal might be largely varied. In the last decade, many studies were carried out for finding stable $\mathrm{BN}$ features that could represent changes of material property. In order to measure the residual stress of materials, Aki Sorsa et al. ${ }^{[3-}$ ${ }^{6]}$ assembled the features including peak information, statistical results, bandwidth, 
entropy and the parameter in $\mathrm{BN}$ profile and then concatenated them to predict the property of materials (e.g. hardness of $18 \mathrm{CrNiMo} 7-6^{[6]}$ ). Liu. et al. ${ }^{[7]}$ compared two models which are multivariable linear regression (MLR) model and BP neural network in applying surface hardness prediction of $12 \mathrm{CrMoV}$ steel plate. $6 \mathrm{BN}$ features (such as peak value, mean value of BN profile) and 5 tangential magnetic field (TMF) features (such as amplitude of the $3^{\text {rd }}, 5^{\text {th }}$ and $7^{\text {th }}$ harmonics) were extracted as the input to the two regression models. In our previous work, ${ }^{[8]}$ we have focused on the feature extraction methods of $\mathrm{BN}$ for hardness prediction. Compared with the conventional $\mathrm{BN}$ features (called isolated features in ${ }^{[8]}$ ), a stable and unified feature that was generated by signal component analysis (i.e. slow feature analysis and discriminant incoherent component analysis) was proposed to predict the hardness of X12m. It could be observed that these mentioned prediction systems are designed to deal with multiple inputs. Therefore, the multi-variable regression method should be adopted in these prediction systems. However, the regression models (e.g. linear regression ${ }^{[7]}$, neural network ${ }^{[9-11]}$, and partial linear regression method ${ }^{[3-4]}$ ) adopted in the previous mentioned methods share the limited capacity. Among different regression tools, the polynomial-based multi-variable regression outperforms other methods in regression accuracy. In NDE research domain, to our best knowledge, it is the first time that the polynomial-based multi-variable regression method is employed to predict the properties of material.

The remainder of this paper is organized as follows: Section II introduces the details of our method. Section III presents experimental setup, results and discussions. Finally, Section IV provides the summary and conclusion.

\section{Methods}

In this section, the $\mathrm{BN}$ features information will be firstly proposed. Then the basic model of multi-variable Chebyshev polynomials regression and two improving algorithms on the polynomial regression will be introduced.

\subsection{Data Preprocessing}

In this study, for each measurement, the detected $\mathrm{BN}$ signal contains 6 complete magnetization loops or $12 \mathrm{BN}$ loops. The first task in the subsection is to remove the incomplete loops and divide the signal into 11 intervals that starts from the same strength of tangential magnetics. Each interval includes one complete magnetization loop. To remove the incomplete loops, the position of the first peak among whole $\mathrm{BN}$ signal is detected as the cut-off point from which the 11 analyzed intervals are extracted. Then in each analyzed BN interval, a moving average window is slide on the signal to filter noise and build the profile. Subsequently, 9 features are extracted, as shown by Figure. 1 and Table 1. With the increasing number of hardness, the qualified relationship between hardness and each chosen feature could be shown in Figure.1. Some features such as peak value show a downward trend whereas some are upward trend such as peak position. In contrasts to the conventional features (i.e. peak information, bandwidth and intercept of tangential magnetic field), the information of sub-peak value and frequency domain were adopted in this paper. According to the literature ${ }^{[6]}$, the hardness of materials is link strongly to the main peak of BN signal profile. Therefore, the sub-peak is employed here as an auxiliary feature for the prediction of hardness. The relationship between sub-peaks and main peak are shown in Figure.2. Besides, the harmonic information of BN includes 
the value of $3^{\text {rd }}, 5^{\text {th }}$ and $7^{\text {th }}$ harmonic points were adopted in this study as mentioned in ${ }^{[7]}$. All the mentioned features are concatenated as the input of our regression algorithm.

Table 1. The selected features in our study

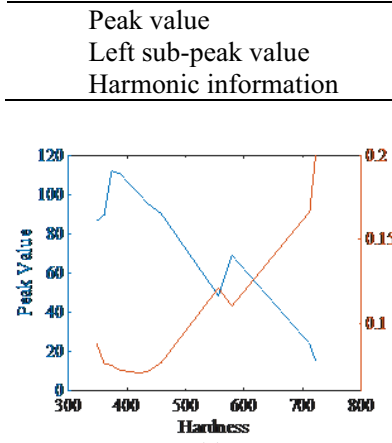

(a)

Peak Position
Right sub-peak value
$75 \%$ bandwidth
$75 \%$ bandwidth
Mean value of $\mathrm{BN}$ profile

Intercept of tangential magnetic field Half bandwidth

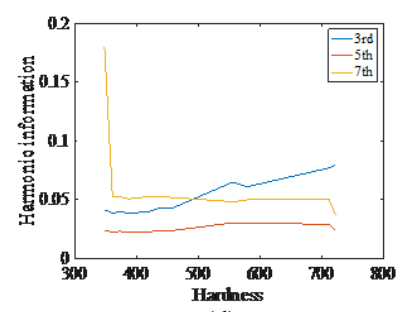

(d)

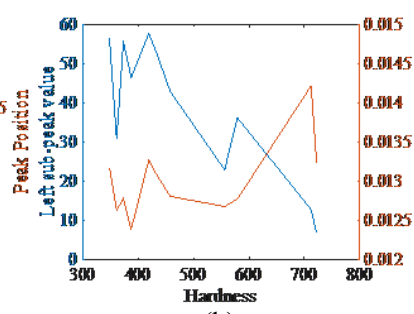

(b)

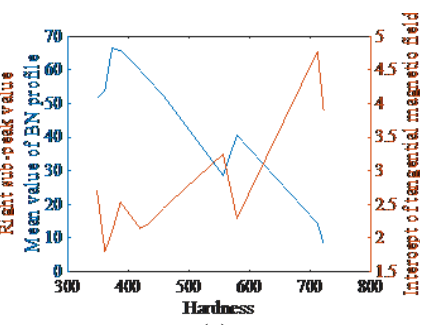

(c)

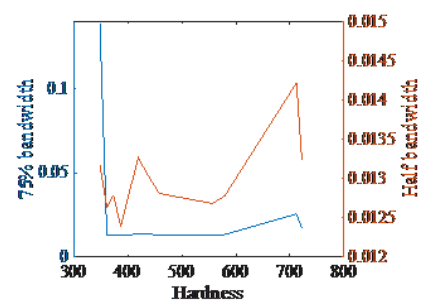

(e)

Figure. 1 Features vary as hardness of X12m increased. (a) Peak value and Peak position, (b) Left sub-peak value and Right sub-peak value, (c) Mean value of BN profile and Intercept of tangential magnetic field, (d) 3rd, 5th and 7th Harmonic information, (e) 75\% bandwidth and Half bandwidth.

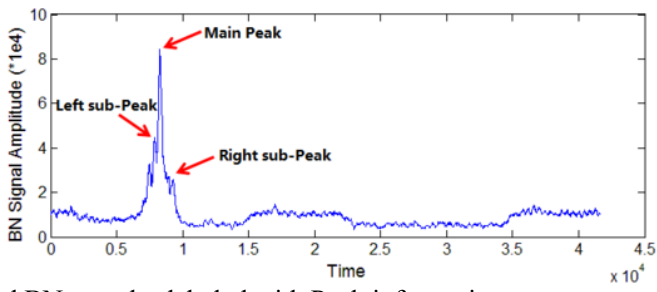

Figure. 2 Two half-period BN samples labeled with Peak information.

\subsection{Introduction to Regression Methods}

Given a variable $x \in(-1,1)$ and the parameter $n \in Z_{0}$, the Chebyshev polynomials of class 2 (i.e. CP-2) $U_{n}(x)$ is defined as:

$$
U_{n}(x)=\frac{\sin ((n+1) \arccos x)}{\sin (\arccos x)}
$$

And the CP-2 could be rewritten as the second-order recursion ${ }^{[15]}$ :

$$
U_{n+2}(x)=2 x U_{n+1}(x)-U_{n}(x) \text { with } U_{0}(x)=1 \text { and } U_{1}(x)=2 x
$$

The first eight terms of CP-2 polynomials are listed in Table 2. Based on the singlevariable CP-2, Zhang Y. et al. ${ }^{[14]}$ further designed the multi-variable format of CP-2 by adopting Bernstein polynomials, which is called multi-variable Chebyshev polynomials regression (MCPR). However, the number of regressed coefficients of MCPR is exponentially proportion to the number of input variables. To overcome the coefficient 
explosion problem, in our work ${ }^{[12]}$, two strategies including feature selection and cascaded regression are designed to alleviate this problem.

Table 2. The first eight CP-2 polynomials

$\begin{array}{cc}U_{0}(x)=1 & U_{4}(x)=16 x^{4}-12 x^{2}+1 \\ U_{1}(x)=2 x & U_{5}(x)=32 x^{5}-32 x^{3}+6 x \\ U_{2}(x)=4 x^{2}-1 & U_{6}(x)=64 x^{6}-80 x^{4}+24 x^{2}-1 \\ U_{3}(x)=8 x^{3}-4 x & U_{7}(x)=128 x^{7}-192 x^{5}+80 x^{3}-8 x\end{array}$

In terms of feature selection idea, a novel feature selection method ${ }^{[13]}$ named "Mutual Information-based Feature Selection with Class-dependent Redundancy" (MIFS-CR) are combined with MCPR to directly reduce the number of the input variables of MCPR. Inspired by the idea that the potential and selected variable equally contribute to the redundancy of mutual information between selected variable and class set, MIFS-CR achieves better performance than the former feature selection methods and the details of MIFS-CR could be found in the related manuscript $t^{[13]}$.

In terms of cascaded regression idea, on the foundation of the cascaded regression and the framework of PLSR ${ }^{[16]}$, our CP-2-based cascaded regression idea could be represented as the following iteration way:

$$
\begin{aligned}
& E_{t}=Z_{t} c_{t}{ }^{T}+E_{t+1} \\
& F_{t}=Z_{t} r_{t}^{T}+F_{t+1}
\end{aligned}
$$

where $t$ is the iteration number, $E_{t}, F_{t}$ are the residual input and target in the former $t-1$ th iteration, $c_{t}, r_{t}$ represent the regression coefficients. As the most important element in (3), $Z_{t}$ is defined as:

$$
Z_{t}=U\left(u_{t}\right) \in R^{N \times n}=\bigcup_{l=1}^{N} \coprod_{j=1}^{n} U_{s_{j}}\left(\left(u_{t}\right)_{l}\right)
$$

where $n$ is the number of CP-2 polynomials, $N$ is the sample number, $u_{t}$ is denoted as the main component of $E_{t}, \amalg$ represents concatenating operator in the column of $Z_{t}$ and $\bigcup$ represents the concatenating operator in the row. In other words, in $t$ th iteration, the residual target $F_{t}$ and input $E_{t}$ is approximated by the single-variable CP-2 regression tool and the variable $u_{t}$ is the main component of residual input $E_{t}$ (i.e. $u_{t}=E_{t} p_{t}$, where $p_{t}$ is the projection vector generated from $\left.E_{t}^{T} F_{t} F_{t}^{T} E_{t}{ }^{[16]}\right)$. And the errors generated from regression tool is treated as the residual target and input for the next iteration $t+1$.

\section{Experiments}

\subsection{Experiment material and data measurements}

Plate samples with the size of $200 \mathrm{~mm} * 60 \mathrm{~mm} * 3 \mathrm{~mm}$ manufactured from case-hardened steel $\mathrm{Cr} 12 \mathrm{MoV}$ (i.e. $\mathrm{x} 12 \mathrm{~m}$ ) were studied in this paper. In the quenching procedure, all tested samples were carburizing case-hardened at $650^{\circ} \mathrm{C}$ for $60 \mathrm{~min}, 850^{\circ} \mathrm{C}$ for $90 \mathrm{~min}$, $1030^{\circ} \mathrm{C}$ for $150 \mathrm{~min}$, and then Nitrogen-cooling for $25 \mathrm{~min}$. In the tempering procedure, the tempering temperature varied between 180 and $720^{\circ} \mathrm{C}$ and times for 210 min which is related to the hardness label of each sample. The properties of $\mathrm{Cr} 12 \mathrm{MoV}$ material and initialization about measurement are shown in Table 3. 
As shown by Figure. 3, the MBN sensor are composed of the excitation coil with 2100 turns winding onto the U-shape ferrite core and the hall sensor next to the surface of sample. The excitation signal was the singular wave with $12 \mathrm{~Hz}$ and $8 \mathrm{~V}$ and generated by LabVIEW. The sampling rate was chosen as $1 \mathrm{MHz}$. And the BN signal was stored with NIPXle multi-channel acquisition card and LabVIEW software. For each sample, the measurement was repeated for 5 times and the $\mathrm{BN}$ signal generated from each repetition contained 12 magnetization loops.

Table 3. The properties of $\mathrm{Cr} 12 \mathrm{MoV}$ material and initialization about measurement

\begin{tabular}{llllllllllll}
\hline Tempering Temperature $\left({ }^{\circ} \mathrm{C}\right)$ & 720 & 700 & 680 & 660 & 640 & 590 & 575 & 550 & 525 & 505 & 180 \\
Averaged Vickers hardness & 348 & 361 & 373 & 387 & 419 & 434 & 458 & 556 & 579 & 712 & 722 \\
\hline
\end{tabular}
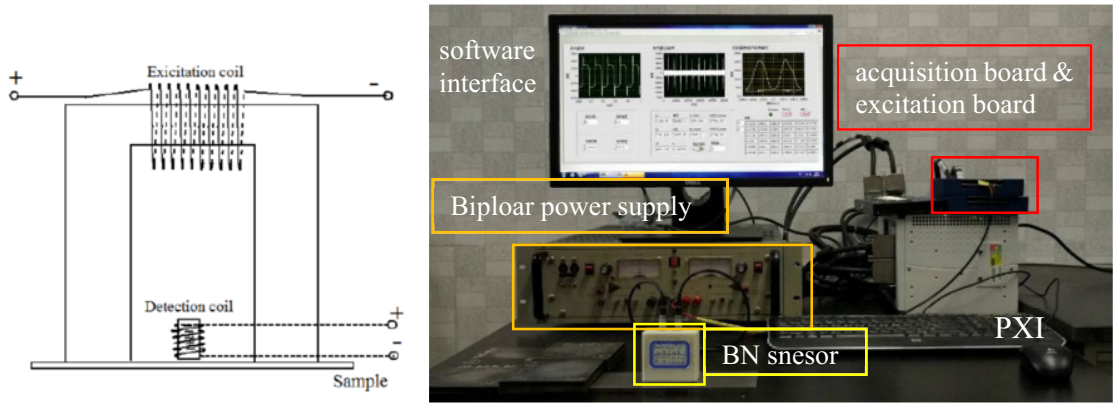

Figre.3 The diagram of sensor settlement.

In terms of hardness prediction experiment, RMSE is used as the criterion for accuracy of prediction, three conventional models and representative methods were compared. These conventional methods include the neural network (i.e. back propagation neural network (BP)), cascaded regression method (i.e. PLSR) and random forests (RF). All algorithms involved in this paper were tested in a 10-cross-validation (CV) way.

\subsection{Results}

The Vickers hardness prediction results are shown by Figure. 4 and the accuracy results about different algorithms are presented by Table 4. According to the Figure. 4(a-c), the scatter points are far away from the base line and imply that the accuracy of the conventional algorithms is unacceptable. This phenomenon could also be found in Table 4. Compared with the results brought by the conventional algorithms, the scatter points of our designed algorithms (shown by Figure.4(d-e)) are closer to the base line and the best performance is achieved by MIFS-CR+CP-2 (i.e. Figure.4(d)). In accordance with Table.4, the RMSE of CP-2-based algorithms is less than other conventional algorithms and the best accuracy (i.e. 0.3629) is achieved by MIFS-CR+CP-2. This high performance could be ascribed to three aspects: Firstly, given a proper order, any nonlinear model could be approximated by an orthogonal polynomials; Secondly, compared with the NN-based model, a specific mathematical equation could be deducted by the polynomials-based model; Thirdly, according to the theory of PCPR, the basic idea of PCPR is to enhance the efficiency with the cost of accuracy ${ }^{[12]}$ and therefore the prediction error is slightly larger than feature-selection-based MCPR method (i.e. MIFS$\mathrm{CR}+\mathrm{CP}-2$ ). Therefore, $\mathrm{CP}-2$ could be ascribed as the main reason for this phenomenon and the enhancement of the prediction accuracy by multi-variable CP-2 regression tool is our main contribution. 
Table 4. The regression accuracy

\begin{tabular}{llllll}
\hline Algorithms & BP & PLSR & RF & MIFS-CR+CP-2 & PCPR \\
\hline$R M S E(H V)$ & 0.5548 & 0.5331 & 0.5151 & $\mathbf{0 . 3 6 2 9}$ & 0.4144 \\
\hline
\end{tabular}

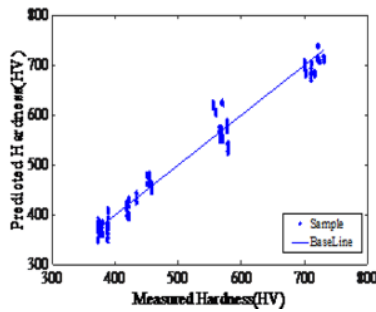

(a)

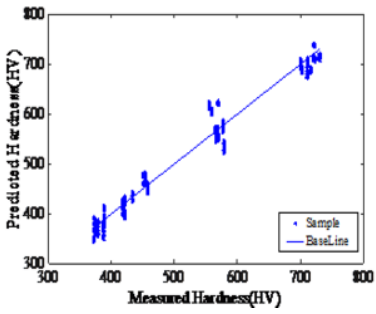

(b)

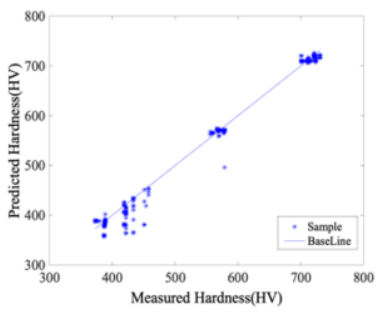

(c)

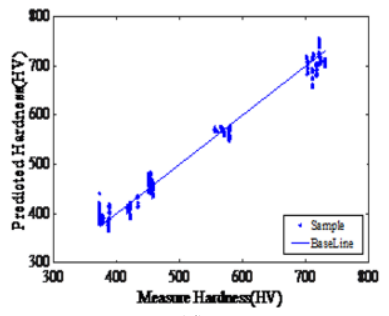

(d)

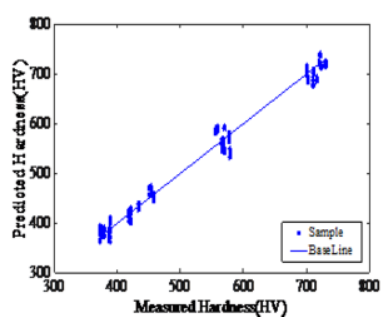

(e)

Figure. 4 Scatterplot of predicted output and the measured output with different algorithms based on Cr12MoV material. (a) BP (b) PLSR (c) RF (d) MIFS-CR+CP-2 (e) PCPR.

\section{Conclusions}

In this paper, the feature-selection-based and cascade-based regression algorithms (i.e. MIFS-CR+CP-2 and PCPR) proposed in our previous study are employed for the purpose of accurate hardness prediction of $\mathrm{X} 12 \mathrm{~m}$ steel by analyzing Barkhausen noise. In the data processing part, conventional and self-designed $\mathrm{BN}$ features are generated. All features are proven that the qualified relationship between hardness and selected feature. In experimental part, the Vickers hardness of $\mathrm{Cr} 12 \mathrm{MoV}$ steel is predicted using the conventional NDE-applied regression tools and our proposed regression algorithms. As shown in the results, the performance of our feature-selection-based regression tool outperforms the other methods. Due to the wide-applied property of regression tools, it could be inferred that CP-2-based regression tools could be applied to solve the hardness prediction problem and have the potential to solve other NDE problems. Therefore, our algorithms can be expected to be further employed in more NDE applications.

\section{References}

[1] Kashefi M, Rafsanjani A, Kahrobaee S, et al. Magnetic nondestructive technology for detection of tempered martensite embrittlement. Journal of Magnetism and Magnetic Materials, 324(2012), 40904093.

[2] Bray D E, Stanley R K. Nondestructive evaluation: a tool in design, manufacturing and service. CRC press, 1996.

[3] Sorsa A, Isokangas A, Santa-aho S, et al. Prediction of residual stresses using partial least squares regression on Barkhausen noise signals. Journal of Nondestructive Evaluation, 33(2014), 43-50. 
[4] Sorsa A, Santa-aho S, Vippola M, et al. A case study of using radial basis function neural networks for predicting material properties from Barkhausen noise, signal Proceedings of 18th Nordic Process Control Workshop, 6p. 2013,3633-3642.

[5] Sorsa A, Leivisk, K, Santa-aho S, et al. An efficient procedure for identifying the prediction model between residual stress and Barkhausen noise. Journal of Nondestructive Evaluation, 32(2013), 341-349.

[6] Sorsa A, Leivisk, K, Santa-aho S, et al. Quantitative prediction of residual stress and hardness in casehardened steel based on the Barkhausen noise measurement. Ndt \& E International, 46(2012), 100-106.

[7] Liu XC, Zhang RH, Wu B, et al. Quantitative Prediction of Surface Hardness in 12CrMoV Steel Plate Based on Magnetic Barkhausen Noise and Tangential Magnetic Field Measurements. Journal of Nondestructive Evaluation, (2018) 37:38.

[8] Li Z, Sun G, He C, et al. Prediction of the hardness of X12m using Barkhausen noise and component T analysis methods. Journal of Magnetism and Magnetic Materials, 478(2019), 59-67.

[9] Perez-Benitez J A, Espina-Hernandez J H, Martínez-Ortiz P. Unwrapping the influence of multiple parameters on the Magnetic Barkhausen Noise signal using self-organizing maps. NDT \& E International, 54(2013): 166-170.

[10] Perez-Benitez J A, Padovese L R. Feature selection and neural network for analysis of microstructural changes in magnetic materials. Expert Systems with Applications, 38(2011), 10547-10553.

[11] Perez-Benitez J A, Padovese L R. Study of the influence of simultaneous variation of magnetic material microstructural features on domain wall dynamics. Journal of Magnetism and Magnetic Materials, 322(2010), 3101-3105.

[12] Z. Li, G. Sun., et al. Multi-variable Regression Methods using Modified Chebyshev Polynomials of Class 2. Journal of Computational and Applied Mathematics, 346 (2019), 609-619.

[13] Zhang Y, Yin Y, Guo D, et al. Cross-validation based weights and structure determination of Chebyshevpolynomial neural networks for pattern classification. Pattern Recognition, 47(2014), 3414-3428.

[14] Wang Z, Li M, Li J. A multi-objective evolutionary algorithm for feature selection based on mutual information with a new redundancy measure. Information Sciences, 307(2015), 73-88.5-7916.

[15] Celant G, Broniatowski M. Interpolation and Extrapolation Optimal Designs V1: Polynomial Regression and Approximation Theory. John Wiley \& Sons, 2016.

[16] Bastien P, Vinzi V E, Tenenhaus M. PLS generalised linear regression. Computational Statistics \& Data Analysis, 48(2005), 17-46. 